\title{
Primordial atmospheric evolution recorded in the Martian mantle
}

\author{
KAVEH PAHLEVAN ${ }^{1}$, LAURA SCHAEFER ${ }^{2}$ AND MARC \\ M HIRSCHMANN ${ }^{3}$ \\ ${ }^{1}$ SETI Institute \\ ${ }^{2}$ Stanford University \\ ${ }^{3}$ University of Minnesota \\ Presenting Author: kpahlevan@seti.org
}

Mars and its primordial atmosphere are thought to have been coeval, having formed via the same process: collision of solidbodies during accretion [e.g., 1]. Indeed, thermal blanketing by the primordial atmosphere may have been central to early thermal evolution and Martian magma ocean formation. However, despite the importance of the primordial atmosphere for early Martian evolution, constraints on its composition and evolution have been elusive. In particular, over the range of oxygen fugacity $\left(\mathrm{fO}_{2}\right)$ that can characterize equilibration with the magma ocean $(\approx \mathrm{IW}-2$ to $\approx \mathrm{QFM})$, the composition of co-existing atmospheres range from reducing $\left(\mathrm{H}_{2}-\mathrm{CO}\right.$-rich) to oxidizing $\left(\mathrm{H}_{2} \mathrm{O}-\mathrm{CO}_{2}\right.$-rich) [2], with important consequences for the subsequent history.

Partial melts sampling the Martian mantle record a range of oxygen fugacities from $\approx$ IW to $\approx$ QFM [e.g., 3], variability whose origin is still unclear. Recently, it has been proposed that $\mathrm{H}_{2}$ outgassing from the Martian magma ocean is responsible for this redox variability [4], since outgassing during magma ocean crystallization is inevitable [e.g., 5] and the redox process is supported by results from experimental petrology [e.g., 6]. Despite the appeal of this process, any $\mathrm{H}_{2}$ outgassed from the Martian magma ocean would presumably be accompanied by heavier species (e.g., CO) and remain gravitationally bound with respect to hydrodynamic escape on dynamical timescales [e.g., 1], continuing to interact with the magma ocean. Accordingly, redox evolution of a Martian magma ocean and primordial atmosphere is expected to be a coupled problem.

Motivated by these questions, we have developed a model of magma ocean-primordial atmosphere evolution to track redox variation arising from crystallization-linked outgassing, magma ocean-atmosphere re-equilibration, and hydrodynamic escape on long (UV-energy-limited) timescales. By coupling the petrology of magma oceans with the physics of atmospheres, we subject the $\mathrm{H}_{2}$ loss hypothesis for the origin of Martian mantle redox variability to a first modeling test.

References: [1] Saito, H. \& Kuramoto, K. (2018) MNRAS 475, 1274-1287 [2] Hirschmann, M.M., (2012) EPSL, 341-344, 48-57 [3] Wadhwa, M. (2008) Rev. Min. Geochem. 68, 493-510 [4] Hirschmann, M.M. (2020) LPSC, Abs. 2221 [5] ElkinsTanton, L. (2008) EPSL, 271, 181- 191 [6] Waters, L. \& Lange, R. (2016) EPSL 447, 48-59. 